\title{
Labyrinthe
}

$10 \mid 2001$

Numéro 10

\section{Activité de la traduction médiévale}

"The Sixth Cardiff Conference on the Theory and Practice of Translation in the Middle Ages ». Université de Saint-Jacques de Compostelle, 19 au 19 juillet 2001.

Jean-Pascal Pouzet

\section{OpenEdition}

Journals

Édition électronique

URL : http://journals.openedition.org/labyrinthe/1200

DOI : $10.4000 /$ labyrinthe. 1200

ISSN : 1950-6031

Éditeur

Hermann

Édition imprimée

Date de publication : 1 octobre 2001

Pagination : 107-109

Référence électronique

Jean-Pascal Pouzet, «Activité de la traduction médiévale », Labyrinthe [En ligne], 10 | 2001, mis en ligne le 07 avril 2006, consulté le 19 avril 2019. URL : http://journals.openedition.org/labyrinthe/1200 : DOI : 10.4000/labyrinthe. 1200

Ce document a été généré automatiquement le 19 avril 2019

Propriété intellectuelle 


\title{
Activité de la traduction médiévale
}

\author{
"The Sixth Cardiff Conference on the Theory and Practice of Translation \\ in the Middle Ages ». Université de Saint-Jacques de Compostelle, 19 au \\ 19 juillet 2001.
}

Jean-Pascal Pouzet

1 En quatre pleines journées, s'est tenue à l'université de Saint-Jacques de Compostelle (Galice) la sixième Conférence internationale sur la Théorie et la Pratique de la Traduction au Moyen Âge. Quelques mots peut-être sont nécessaires pour présenter l'originalité et l'importance des "Cardiff Conferences». Nées à la fin des années 1980 sous l'impulsion (notamment) de Roger Ellis (University of Cardiff) et René Tixier (université de Toulouse I), ces rencontres galloises optent depuis huit ans pour un heureux nomadisme européen : trois éditions à Cardiff (1987, 1989 et 1991), puis une à Conques (France, 1993) et une autre à Göttingen (Allemagne, 1996) ont donné lieu à de fructueux échanges, dont les publications respectives des actes sont le reflet. Plus connue des médiévistes sous l'étiquette "The Medieval Translator " (qui lui vient du nom familier de ces publications), la structure d'ensemble n'est pas une association traditionnelle au sens juridique du terme. Elle est plutôt le rassemblement de chercheurs de nombreux pays, que rapproche dans leur travail un intérêt aigu pour l'activité de traduction au Moyen Âge, sans exclusive de langue, de pays ni d'approche, et auquel la Conférence internationale donne une forme aussi triennale (en moyenne) qu'amicale.

2 La sixième Conférence est le fruit d'une collaboration efficace et sympathique entre Rosalynn Voaden (Department of English, Arizona State University) et Teresa Sánchez Roura (Departamento de Filoloxia Inglesa, Universidade de Santiago de Compostela), avec la précieuse assistance d'un comité local constitué par les médiévistes anglicistes de l'université d'accueil. Sous ces auspices, les 65 orateurs retenus ont eu le loisir de s'exprimer dans l'une des trois langues officielles (anglais, français, espagnol), mais c'est de loin l'anglais qui a été le plus représenté (seulement 5 communications en espagnol et 4 en français). Une telle prépondérance de l'anglais a des raisons historiques. Par la nature des spécialités scientifiques de ses fondateurs et de ses premiers participants, les origines du projet font la part belle à l'activité traductrice ayant pour « langue d'arrivée » 
l'anglais du Moyen Âge, qu'il s'agisse du vieil ou du moyen anglais, et que la langue de départ soit le latin ou toute langue vernaculaire de l'Europe médiévale. De fait, les chercheurs présents appartiennent pour l'essentiel au monde universitaire anglo-saxon, et consacrent leurs travaux à des textes où la langue anglaise tient une place importante. Si cette tendance est confirmée cette année encore pour la grande majorité des communications, la rencontre n'en est pas moins dès l'origine ouverte aux autres langues dont résonne le Moyen Âge occidental, et dont la présence relative s'est malgré tout renforcée à Compostelle. C'est ainsi par exemple qu'un atelier intitulé "Traduction et Orient » a regroupé des communications portant sur les traductions de et vers le grec et l'arabe, par rapport au latin.

Il est impossible de donner ici un aperçu des nombreuses tendances critiques qui ont sous-tendu cette rencontre. Les aspects "théorique » et "pratique » ont été explorés conjointement dans chaque communication ; à l'évidence, les deux éléments qui figurent dans l'intitulé des «Cardiff Conferences » ne déchirent pas la traduction entre d'artificielles et improbables polarités, mais invitent plutôt à conjuguer theoria et praxis dans un fructueux va-et-vient, pensé dans des termes aussi appropriés que possible, selon la nature des textes considérés. Dans leur ensemble, les chercheurs ont manifesté leur attachement à la confrontation entre les considérations proprement médiévales (telles qu'elles peuvent notamment se recueillir, de manière fragmentaire, à la lecture des nombreux prologues d'auteurs) et les réflexions modernes sur le traduire médiéval. Les intitulés respectifs des ateliers étaient délibérément présentés sous la forme « Traduction et... ", dans un double souci de lisibilité et d'harmonisation dans l'organisation. Quelquesuns des titres retenus peuvent rendre compte des axes sous lesquels les communications diverses ont été fédérées: "Traduction et humanisme ", "Traduction et philologie ", " Traduction et iconographie ", "Traduction et manuscrits ", "Traduction et mécénat ", "Traduction et hagiographie ». Nul doute que la présence relative de ces différents contenus peut renseigner sur leur représentativité institutionnelle dans les études médiévales en Europe et dans le monde anglo-saxon. Ainsi, "Traduction et romance médiéval » et "Traduction et Chaucer » ont fait chacun l'objet de deux sessions, étant donné le nombre des communications présentées : le romance et Chaucer demeurent bel et bien les domaines les plus populaires des études médiévales anglaises. A contrario, les deux seules communications portant sur le vieil anglais se sont trouvées regroupées sous le vocable « Traduction et langues germaniques vernaculaires ».

Deux exemples de communication donneront une petite image de la diversité des échanges. Si "traduction" a été pris essentiellement dans un sens «textuel », l'atelier "Traduction et iconographie » a permis d'explorer les relations de «transport de sens » entre texte et image. Le professeur Takami Matsuda (Université de Keio, Japon) a ainsi offert une superbe analyse des rapports entre une anthologie illustrée de textes de dévotion en moyen anglais (ca. 1470) et le programme iconographique qu'il comporte, montrant de manière convaincante combien l'étude du MS Londres, British Library, Additional 37049 ne saurait plus longtemps se couper d'une interaction fort complexe. Dans l'atelier «Traduction et théologie vernaculaire » (auquel a participé l'auteur de ces lignes), le professeur A. J. Minnis (Université de York, Grande-Bretagne), auteur de nombreux travaux sur la théorie littéraire médiévale et les sources d'auctoritas ${ }^{1}$ a présenté un fascinant dossier concernant Walter Brut, théologien contestataire de la fin du XIV siècle, relatif à la question de l'administration du sacrement de l'Eucharistie par 
une femme. C'était là rappeler de façon magistrale combien le corps (masculin, féminin) est le lieu d'autorité premier en matière de « translation » religieuse.

5 Le succès de cette sixième édition tient aussi à l'excellent accueil qu'elle a réservé aux tout jeunes chercheurs. Ces rencontres très appréciées ne peuvent qu'inviter à un accroissement continu des membres, et donc de leurs sujets de recherche, pour continuer à offrir un espace ouvert et compétent de recherche sur la traduction médiévale.

\section{NOTES}

1. On retiendra : A. J. Minnis, Medieval Theories of Authorship. Scholastic Literary Attitudes in the Later Middle Ages, $2^{\mathrm{e}}$ edition, Aldershot, 1988.

\section{AUTEUR}

\section{JEAN-PASCAL POUZET}

Ancien élève de l'École Normale Supérieure, agrégé d'anglais, Jean-Pascal Pouzet est allocataire-moniteur à l'Université Paris IV-Sorbonne. Il prépare une thèse sur les traductions versifiées de la Bible dans la littérature moyen anglaise ( $\mathrm{XIII}^{\mathrm{e}}-\mathrm{XV}^{\mathrm{e}} \mathrm{s}$.). En parallèle, le travail présenté ici est l'écho d'une recherche qu'il vient de commencer, portant sur les modes d'expression de la théologie vernaculaire dans les textes en prose. 